\title{
Transmission Kikuchi Diffraction of the Thermally Grown Oxide on Grain-refined NiAl-Zr
}

\author{
Rachel White $^{1}$ and Mark Weaver ${ }^{1}$ \\ 1. The University of Alabama Metallurgical and Materials Engineering, Tuscaloosa, AL.
}

Grain refinement of high temperature oxidation resistant materials has been shown to expedite oxide phase transformations and to improve spallation resistance [1]. However, very little work has been done to investigate the resulting microstructure in detail. The present study correlates the oxidation mass change behavior to the oxide microstructural features of grain-refined NiAl-0.09Zr and extruded NiAl-0.05Zr (at.\%) using secondary electron microscopy (SEM), transmission electron microscopy (TEM), and transmission kikuchi diffraction (TKD).

Grain refinement was achieved by DC magnetron sputter coating from a NiAl-0.09Zr target. Hot extruded NiAl-0.05Zr substrates were used as control specimens. Samples were oxidized in an $80 \%$ argon $-20 \%$ oxygen atmosphere. TKD parameters are listed in Table 1. Ten bands were used for orientation analyses, while 12 bands were used for phase identification, coupled with a six neighbor clean up. The sample thickness was estimated to be $\sim 100 \mathrm{~nm}$, as thinner samples produced a very weak signal, even with a lower accelerating voltage.

Thermogravimetric oxidation (Figure 1) showed the sputter coated sample to have the earliest transition to steady state oxidation, but with a steady state oxidation rate constant that was comparable to other NiAl$\mathrm{Zr}$ materials. The exception was the extruded NiAl-0.05Zr, which exhibited a steady state rate constant that was almost an order of magnitude lower. The steady state oxidation rate is a function of oxide growth, which can occur as the oxide thickens or as it regrows after spallation. The extruded and grain-refined sample both exhibited excellent spallation resistance.

TEM bright field imaging was used to study the oxide morphology, as the $\theta$ phase grows in very fine equiaxed grains and/or long whiskers, while the $\alpha$ phase nucleates at the metal-oxide interface as larger equiaxed or columnar grains [2]. The transition from transient to steady state oxidation is marked by the formation of $\alpha$ grains, which owing to their low diffusivity protect the underlying metal. TEM showed that by 5 hours of oxidation the grain-refined sample had already grown a continuous layer of $\alpha$ at the metal-oxide interface, compared with the extruded sample, which only showed a couple of $\alpha$ grains at the metal-oxide interface. The grain-refined oxide showed a three-layer structure of $\alpha$ grains on the bottom, small oxide grains and high atomic number $(Z)$ contrast precipitates in the middle, and $\theta$ whiskers on top. This three-layer structure was retained through 50 hours of oxidation, by which time the top whisker layer had transformed to $\alpha$.

TKD painted a clearer picture of the oxide grain structure (Figure 2). The band contrast map shows $<100$ $\mathrm{nm} \alpha$ and $\mathrm{ZrO}_{2}$ grains in the middle oxide layer. $\mathrm{ZrO}_{2}$ has a stronger signal and is a lighter grey color. Both monoclinic and tetragonal $\mathrm{ZrO}_{2}$ phases were confirmed by the TKD phase map. Columnar $\alpha$ grains in the bottom layer measure $0.3 \mu \mathrm{m}$ in diameter and show a random texture, implying inward growth. The top layer contains an $\alpha$ grain approximately $3 \mu \mathrm{m}$ in diameter that shows over $10^{\circ}$ of misorientation and no low angle grain boundaries; this morphology is observed in the literature as the result of the $\theta$ - $\alpha$ phase transformation and is correlated with low diffusion rates [3]. 
TKD coupled with TEM bright field imaging informs the oxide formation mechanism. Zr is known to diffuse rapidly to the free surface at the onset of oxidation and it has been shown to limit outward diffusion of aluminum through $\alpha-\mathrm{Al}_{2} \mathrm{O}_{3}$ scales [4]. It is proposed that the quick diffusion of $\mathrm{Zr}$ to the original metalgas interface resulted in decreased Al diffusion outward. This ended transient whisker growth early and resulted in an inward growing bottom oxide layer. The implications of a fine grained oxide scale include improved spallation resistance, but also increased oxide growth rates as oxide grain boundaries are fast diffusion pathways for oxide-forming species [3]. High grain boundary misorientation is also thought to increase oxide growth rates, however further study is needed.

[1] F Wang, Oxidation of Metals 48(3-4) (1997), p. 215.

[2] P Hou, A Paulikas and B Veal, Materials at High Temperatures 22(3-4) (2005), p. 535.

[3] G Rybicki and J Smialek, Oxidation of Metals 31(3-4) (1989), p. 275.

[4] B Pint, J Martin and L Hobbs, Oxidation of Metals 39(3-4) (1993), p. 167.

\begin{tabular}{|l|l|}
\hline Instrument & JEOL 7000 FEG-SEM \\
\hline Software & Oxford EBSD \\
\hline Working Distance & $2.7 \mathrm{~mm}$ \\
\hline Sample Tilt & $0^{\circ}$ \\
\hline Accelerating Voltage & $30 \mathrm{keV}$ \\
\hline Probe Current & Setting of 16 out of 18 \\
\hline Step Size & $13.5 \mathrm{~nm}$ \\
\hline Camera Binning & $2 \mathrm{x} 2$ \\
\hline Camera Gain & High \\
\hline Dwell Time per Frame & Approx. $20 \mathrm{~ms}$ \\
\hline Frame Averaging & 2 \\
\hline
\end{tabular}

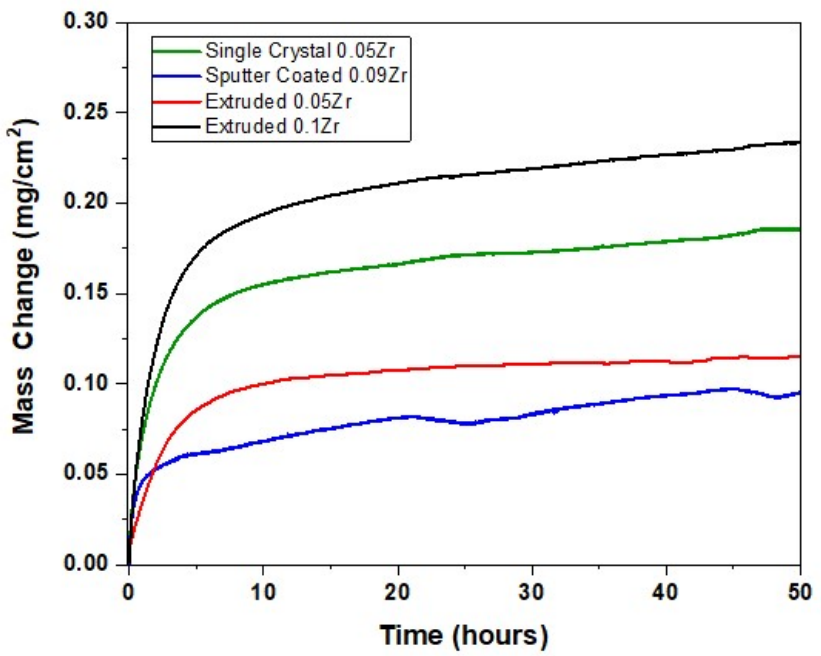

Figure 1. TKD microscope parameters and isothermal mass change curve of single crystal, extruded, and grain-refined samples through 50 hours of oxidation at $1000^{\circ} \mathrm{C}$
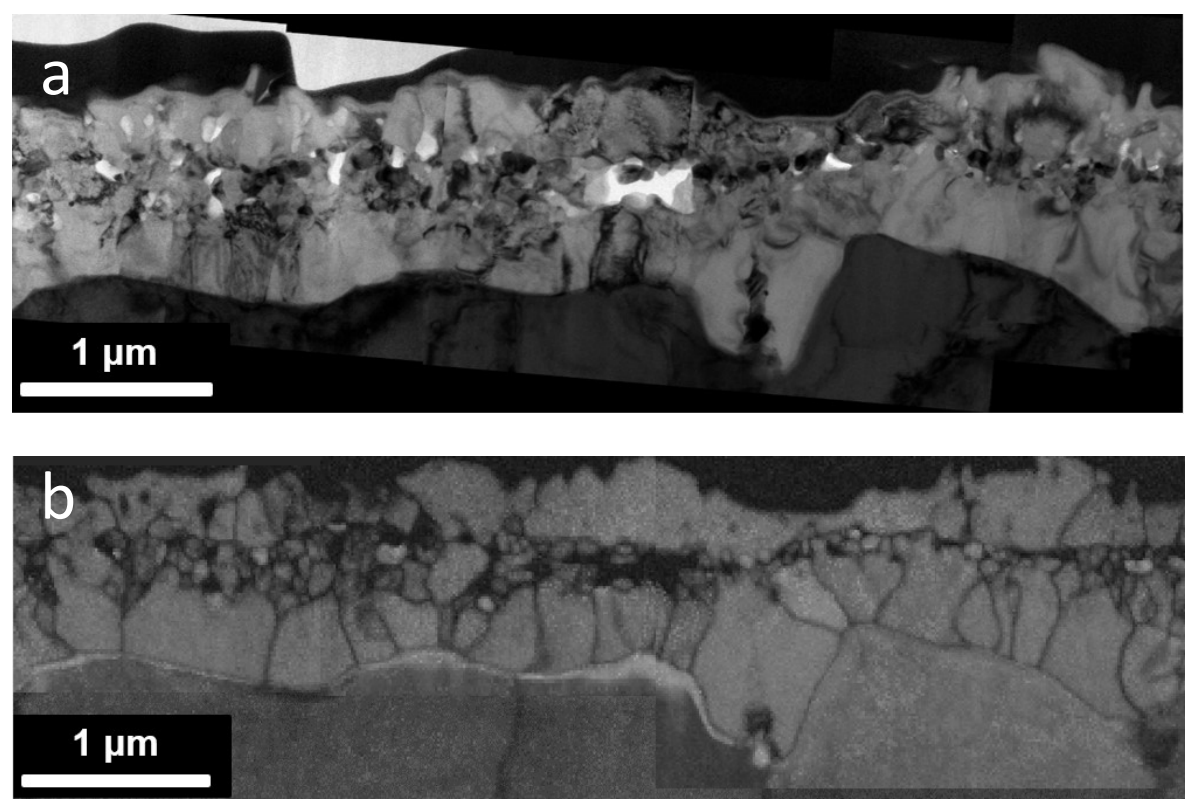

Figure 2. Grain-refined oxide after 50 hours of oxidation: a) TEM bright field, and b) TKD band contrast map showing grain size and morphology of the three-layer oxide. 